\title{
Dynamical boundary of a self-similar set
}

\author{
by
}

\author{
Manuel Mor án (Madrid)
}

\begin{abstract}
Given a self-similar set $E$ generated by a finite system $\Psi$ of contracting similitudes of a complete metric space $X$ we analyze a separation condition for $\Psi$, which is obtained if, in the open set condition, the open subset of $X$ is replaced with an open set in the topology of $E$ as a metric subspace of $X$. We prove that such a condition, which we call the restricted open set condition, is equivalent to the strong open set condition. Using the dynamical properties of the forward shift, we find a canonical construction for the largest open set $V$ satisfying the restricted open set condition. We show that the boundary of $V$ in $E$, which we call the dynamical boundary of $E$, is made up of exceptional points from a topological and measure-theoretic point of view, and it exhibits some other boundary-like properties. Using properties of subselfsimilar sets, we find a method which allows us to obtain the Hausdorff and packing dimensions of the dynamical boundary and the overlapping set in the case when $X$ is the $n$-dimensional Euclidean space and $\Psi$ satisfies the open set condition. We show that, in this case, the dimension of these sets is strictly less than the dimension of the set $E$.
\end{abstract}

1. Introduction. A self-similar set is a set that can be decomposed into subsets which are similar copies of the whole set. Self-similar sets like the Cantor set, the Koch curve or the Sierpiński gasket are amongst the first known examples of fractal sets. The basic ideas leading to the analysis of self-similar sets were originated in 1946 by P. A. P. Moran [13], and developed by Mandelbrot [8] and Hutchinson [5]. A considerable volume of research in the last two decades has focused on these sets (see [9] for some references). Self-similar sets are a canonical type of fractal sets. Their analysis has been relevant for the development of many tools of geometric measure theory and they have also a wide range of applications to various empirical sciences (see [8] and [4]).

1991 Mathematics Subject Classification: 28A78, 28A80.

Key words and phrases: self-similar sets, Hausdorff dimension, open set condition.

Research partially supported by the Dirección General de Investigación Científica y Técnica, PS95-0193. 
In the rest of this section we describe the self-similar constructions we deal with, we give some of their basic properties and we discuss some consequences of the results of the paper. Section 2 is devoted to the analysis of the restricted open set condition and to the analysis of the general properties of the dynamical boundary, and in Section 3 we analyze the fractal dimension of the dynamical boundary and the overlapping set.

1.1. Self-similar set generated by a finite system of similitudes. Given a finite set $\Psi=\left\{\psi_{1}, \ldots, \psi_{m}\right\}$ of surjective and contracting similarities of a complete metric space $X$, there exists a unique compact subset $E \subseteq X$, which we call the self-similar set generated by $\Psi$, such that $S \Psi(E)=E$, where $S \Psi$ is the set mapping defined by

$$
S \Psi(Y)=\bigcup_{i=1}^{m} \psi_{i}(Y), \quad Y \subseteq X .
$$

The proof of this fact for self-similar sets in $\mathbb{R}^{n}$ can be seen in [5]. It depends on the completeness of the set of non-empty compact subsets of $\mathbb{R}^{n}$ endowed with the Hausdorff metric, which also holds if the embedding space is any complete metric space, as in our case. From now onwards we assume that we are given a system $\Psi$ of surjective and contracting similitudes of $X$ and a self-similar set $E$ as defined above.

REMARK 1.1. A useful consequence of the uniqueness of $E$ is the following: Let $K$ be a non-empty compact subset of $X$ such that $S \Psi(K) \subseteq K$. If $S \Psi^{k}$ denotes the $k$ th iterate of the set mapping $S \Psi$, then

$$
\bigcap_{k=1}^{\infty} S \Psi^{k}(K)=E \subseteq K .
$$

This is easily seen by checking that $S \Psi\left(\bigcap_{k=1}^{\infty} S \Psi^{k}(K)\right)=\bigcap_{k=1}^{\infty} S \Psi^{k}(K)$.

1.2. Space of codes of a self-similar set. The self-similar set $E$ is the image of the space of codes $\mathbb{M}=M^{\infty}=M \times M \times \ldots$, with $M=\{1, \ldots, m\}$, under the projection mapping $\pi: \mathbb{M} \rightarrow \mathbb{E}$ given by

$$
\pi(\mathbf{i})=\bigcap_{k=1}^{\infty} \psi_{\mathbf{i}(k)}(E), \quad \mathbf{i}=i_{1} i_{2} \ldots \in \mathbb{M},
$$

where $\mathbf{i}(k)$ denotes the curtailment $i_{1} \ldots i_{k} \in M^{k}$ of $\mathbf{i}$ and, given $\mathbf{j} \in M^{k}, \psi_{\mathbf{j}}$ denotes the similitude $\psi_{j_{1}} \circ \ldots \circ \psi_{j_{k}}$. We abbreviate the sets $\psi_{\mathbf{j}}(E), \mathbf{j} \in M^{k}$, $k \in \mathbb{N}$, to $E_{\mathbf{j}}$. We also write $r_{\mathbf{i}}$ for the contraction ratio of the similitude $\psi_{\mathbf{i}}$, $\mathbf{i} \in M^{k}, k \in \mathbb{N}$.

1.3. Hausdorff measures and the open set condition. Given $s>0$, the Hausdorff $s$-dimensional measure of a subset $Y \subseteq X$ is defined by 


$$
H^{s}(Y)=\lim _{\delta \rightarrow 0} \inf \left\{\sum_{i}\left|B_{i}\right|^{s}\right\}
$$

where the infimum is taken over the countable covers of $Y$ by open balls with diameter smaller than $\delta$, and where $|\cdot|$ stands for diameter. We write $\operatorname{dim} Y$ for the Hausdorff dimension of a subset $Y \subseteq X$, i.e. $\operatorname{dim} Y=$ $\inf \left\{s: H^{s}(Y)=0\right\}$ (see $[2,9]$ for more details on Hausdorff measures and dimension).

We say that $\Psi$ (or $E$ ) satisfies the open set condition (O.S.C.) if there exists a non-empty bounded open set $\mathcal{O} \subseteq X$ such that

(i) $S \Psi(\mathcal{O}) \subseteq \mathcal{O}$,

$$
\text { (ii) } \psi_{i}(\mathcal{O}) \cap \psi_{j}(\mathcal{O})=\emptyset \text { if } i \neq j \text {. }
$$

If also $E \cap \mathcal{O} \neq \emptyset$, then we say that $\Psi$ satisfies the strong open set condition (S.O.S.C.).

Assume that $X=\mathbb{R}^{n}$. It is known $[13,5]$ that if the O.S.C. holds for $\Psi$, then $\operatorname{dim} E=s$ for the unique root $s$ of the equation $\varphi(x)=1$ with $\varphi(x)=\sum_{i=1}^{m} r_{i}^{x}$, and for this value of $s$, we have $0<H^{s}(E)$. A. Schief [14] showed that the O.S.C. is then equivalent to $H^{s}(E)>0$. If the O.S.C. holds with $\mathcal{O}$ as the open set, it may happen that $E \subseteq \partial \mathcal{O}$ ( $\partial \mathcal{O}$ denotes the boundary of $\mathcal{O}$ ). A. Schief [14] also showed that if the O.S.C. holds for some open set, then so does the S.O.S.C. Other interesting separation conditions have been shown to be equivalent to the O.S.C. in the Euclidean case (see [1]).

The equivalence of the O.S.C., the S.O.S.C. and the positiveness of $H^{s}(E)$ still holds in complete metric spaces which are $\beta$-spaces $[16,15]$. In the general case when $X$ is a complete metric space, the situation is quite different. In this setting, the O.S.C. does not imply the S.O.S.C. A. Schief [15] has shown that the S.O.S.C. is still relevant, since it implies that the Hausdorff dimension of $E$ coincides with its similarity dimension. In the quoted reference an example of a self-similar set is shown satisfying the O.S.C. whose Hausdorff dimension decreases to zero, which shows that the O.S.C. may be rather meaningless in complete metric spaces which are not $\beta$-spaces. See [15] for a complete discussion of the logical links among the different separation conditions for self-similar sets in that setting. In Section 2 we focus on finding a canonical way to construct a separating set.

The overlapping set $O \subseteq E$ is defined by $O=\bigcup_{i \neq j} E_{i} \cap E_{j}$. When the O.S.C. holds, to know an upper bound for the dimension of the overlapping set is useful for the analysis of relevant subsets of $E$, for instance, for the analysis of the multifractal components. In [10] it is shown that some results holding in the non-overlapping case can be extended, when $\Psi$ satisfies the O.S.C., to subsets of $E$ with dimension greater than that of the overlapping set, and other results stand for subsets of dimension greater than that of 
the dynamical boundary of $E$ (see Section 1.4 for a definition). In Section 3 we show that the dimension of the dynamical boundary, and the dimension of the overlapping set, of a self-similar set in $\mathbb{R}^{n}$ satisfying the O.S.C. is strictly smaller than the similarity dimension (see Theorem 3.3).

Assume that $\Psi$ satisfies the O.S.C. Let $\operatorname{adh}(Y), Y \subseteq X$, denote the closure of $Y$. From the continuity of $\psi_{i}, i \in M$, together with property (i) in (1) it follows that $S \Psi(\operatorname{adh}(\mathcal{O})) \subseteq \operatorname{adh}(\mathcal{O})$ so, by Remark 1.1, we see that $E \subseteq \operatorname{adh}(\mathcal{O})$, hence $E_{i} \subseteq \operatorname{adh}\left(\psi_{i}(\mathcal{O})\right), i \in M$. Therefore we have

$$
O \subseteq \bigcup_{i \neq j} \operatorname{adh}\left(\psi_{i}(\mathcal{O})\right) \cap \operatorname{adh}\left(\psi_{j}(\mathcal{O})\right) .
$$

Since $\psi_{i}(\mathcal{O}) \subseteq X-\psi_{j}(\mathcal{O})$ for $i \neq j$, we see that

$$
\begin{aligned}
\psi_{i}(\mathcal{O}) & =\operatorname{int}\left(\psi_{i}(\mathcal{O})\right) \subseteq \operatorname{int}\left(X-\psi_{j}(\mathcal{O})\right) \\
& =X-\operatorname{adh}\left(\psi_{j}(\mathcal{O})\right) \subseteq X-\partial\left(\psi_{j}(\mathcal{O})\right),
\end{aligned}
$$

which shows that $\psi_{i}(\mathcal{O}) \cap \partial\left(\psi_{j}(\mathcal{O})\right)=\emptyset$ for $i \neq j$. Therefore we may write

$$
\begin{aligned}
O & \subseteq \bigcup_{i \neq j}\left[\partial\left(\psi_{i}(\mathcal{O})\right) \cup \psi_{i}(\mathcal{O})\right] \cap\left[\partial\left(\psi_{j}(\mathcal{O})\right) \cup \psi_{j}(\mathcal{O})\right] \\
& =\bigcup_{i \neq j} \partial\left(\psi_{i}(\mathcal{O})\right) \cap \partial\left(\psi_{j}(\mathcal{O})\right) .
\end{aligned}
$$

From this it easily follows that

$$
O \cap \psi_{i}(\mathcal{O})=\emptyset, \quad i \in M .
$$

Assume that a non-empty open set $V \subseteq E$ satisfies

$$
\begin{aligned}
& \text { (i) } S \Psi(V) \subseteq V \text {, } \\
& \text { (ii) } \psi(V) \cap O=\emptyset \text { for all } \psi \in \Psi \text {. }
\end{aligned}
$$

In this case we say that $\Psi$ satisfies the restricted open set condition (R.O.S.C.) for the open set $V$. If the S.O.S.C. holds for an open set $\mathcal{O}$, then it is easy to see using (3) that the R.O.S.C. holds for $E \cap \mathcal{O}$. We show in Theorem 2.3 that both separation conditions are indeed equivalent, but to derive the S.O.S.C. from the R.O.S.C. is not trivial, since care is needed to extend the open set satisfying the R.O.S.C. to an open set in $X$ satisfying the S.O.S.C. If the R.O.S.C. holds for a set $W$ then $W=E \cap \mathcal{O}$ for some set $\mathcal{O}$ open in $X$, but even in the case where $S \Psi(\mathcal{O}) \subseteq \mathcal{O}, \psi_{i}(\mathcal{O}) \cap \psi_{j}(\mathcal{O})$ could be non-empty for some pair $\psi_{i}, \psi_{j}$ of similitudes in $\Psi$, as the following example, due to an anonymous referee, shows: Consider a Sierpiński triangle $E$ with side length 1 generated by two similitudes of contraction ratio $1 / 2$, and a third similitude of contraction ratio $1 / 4$ with fixed point at some vertex of the horizontal side of $E$, and let $\mathcal{O}$ be the interior of a triangle with a vertex being the top vertex of $E$, and whose horizontal side is the horizontal side 
of $E$ extended by 1 in both directions. Then the sets $W:=E \cap \mathcal{O}$ and $\mathcal{O}$ are in the situation described.

From condition (ii) of (4) it follows that $\psi_{i}(V) \cap \psi_{j}(V)=\emptyset$ if $i \neq j$, $i, j \in M$. So, a subset of $E$ satisfying the R.O.S.C. also satisfies conditions (1) required for an open subset of $X$ for which the O.S.C. holds. It is easy to see that property (ii) of (4) may fail to hold for a non-empty subset open in $E$ which has properties (i) and (ii) of (1) (take as $V$ the set $[0,1)$ in the standard construction of the unit interval as a self-similar set generated by two similitudes with contraction ratios $1 / 2$ ). We do not know, however, if from the assumption that conditions (i) and (ii) of (1) hold for a non-empty set $V$ open in $E$ it follows that the R.O.S.C. holds for some set open in $E$.

1.4. The shift mapping. The possibility of obtaining useful information on the measure-theoretic properties of $E$ from an analysis of the dynamical properties of the geometric shift (see below for a definition) has been shown in [11] and [12]. In this paper we use topological properties of the dynamics of the geometric shift to construct the largest open set satisfying the R.O.S.C.

The code shift mapping $\sigma: \mathbb{M} \rightarrow \mathbb{M}$ is defined on $\mathbb{M}$ by $\sigma\left(i_{1} i_{2} \ldots\right)=$ $i_{2} i_{3} \ldots$ If $O=\emptyset$, the code shift can be projected onto $E$, thus yielding a geometric shift $T: E \rightarrow E$, given by $T(x)=\pi \circ \sigma \circ \pi^{-1}(x)$. If $O \neq \emptyset$, this last formula defines a set-valued rather than a point-to-point mapping, since $\pi^{-1}(x)$ is, in general, a subset of $\mathbb{M}$. We also write $T(Y)=\pi \circ \sigma \circ \pi^{-1}(Y)$ for subsets $Y \subseteq E$.

Observe that if $x \in T(Y)$ then $x=\pi \circ \sigma(\mathbf{i})$ for some $\mathbf{i} \in \mathbb{M}$ with $\pi(\mathbf{i}) \in Y$, and $\psi_{i_{1}}(x)=\pi(\mathbf{i})$. Conversely, assume that $\psi_{i}(x) \in Y$ with $x \in E$ and $i \in M$. Then $x=\pi(\mathbf{i})$ for some $\mathbf{i} \in \mathbb{M}$, and we have

$$
x=\pi \circ \sigma\left(i i_{1} i_{2} \ldots\right) \in \pi \circ \sigma \circ \pi^{-1}\left(\psi_{i}(x)\right) \subseteq T(Y) .
$$

Therefore

$$
x \in T(Y) \text { if and only if } \quad \psi(x) \in Y \text { for some } \psi \in \Psi .
$$

We will frequently use this fact throughout the text.

The shift orbit $\mathbf{O}(Y)$ of a set $Y \subseteq E$ is defined by $\mathbf{O}(Y)=\bigcup_{i=0}^{\infty} T^{i}(Y)$, with $T^{0}(Y)=Y$. We show in Section 2 that the R.O.S.C. holds if and only if the set $G:=\operatorname{adh}(\mathbf{O}(T(O)))$, which we call the dynamical boundary of $E$, satisfies $E-G \neq \emptyset$, and then $V:=E-G$ is the largest set satisfying the R.O.S.C. (see Remark 2.2 and Theorem 2.3). We also analyze in that section the conditions under which $G$ can be obtained in the simplified form $\bigcup_{i=1}^{q} T^{i}(O)$ for finite $q$ (see Example 2.6, Theorem 2.7, and Proposition 2.8).

There are a number of reasons to regard the set $G$ as a boundary of a self-similar set. First, it is the topological boundary of the largest set satisfying the R.O.S.C. Also it is contained in the topological boundary of any open subset of $X$ satisfying the O.S.C. (see Lemma 2.1). If $E$ has a 
non-empty interior in $X$, then $G$ is the boundary of $E$ (see Proposition 2.8). The points of $G$ have an exceptional behaviour from the following measuretheoretic point of view: Let $\mathbf{p}=\left(p_{i}\right)_{1<i<m}$ be a probability vector, and let $\nu_{\mathbf{p}}=\mathbf{p} \times \mathbf{p} \times \ldots$ be the infinite-fold product probability measure on $\mathbb{M}$ obtained by regarding $\mathbf{p}$ as a probability measure on $M$ given by $\mathbf{p}(i)=p_{i}$, $i \in M$. The measure $\mu_{\mathbf{p}}=\nu_{\mathbf{p}} \circ \pi^{-1}$ defined as the projection of $\nu_{\mathbf{p}}$ on $E$ under $\pi$ is called a self-similar measure in the literature (self-similar measures were introduced in [5]). We say that $\mu_{\mathbf{p}}$ is non-degenerate if $p_{i}>0$ for all $i \in M$. A straightforward adaptation of Theorem 1 of [11] shows that, if $\mu_{\mathbf{p}}$ is non-degenerate and $\Psi$ satisfies the S.O.S.C., then $\mu_{\mathbf{p}}(G)=0$.

We call a subset $Y \subseteq E T$-invariant if $T(Y) \subseteq Y$. The set $G$ is $T$ invariant (see Lemma 2.1). If $y \in T(x)$ for some $x \in E$, then $y=\psi^{-1}(x)$ for some $\psi \in \Psi$, so all "branches" of $T$ are expanding similitudes and, in this sense, the dynamics of the set mapping $T$ is a repelling dynamics on $T$ invariant subsets of $E$. These properties are also shared by a pair $\left(J, f^{-1}\right)$, where $f$ is a rational map in the complex plane and $J$ is its associated Julia set.

We remark that for self-similar sets with $G$ a finite set, Brownian motions are analyzed in [6] and the Laplacian operator is introduced in [7]. In this case the self-similar set is called post-critically finite, a concept which comes from the theory of Julia sets.

2. The restricted open set condition. We denote by $U(x, r)$ the open ball of $X$ centered at $x$ with radius $r$. Given $Z \subseteq X$ the notation $U_{Z}(x, r)$ stands for an open ball in the metric subspace $Z$, i.e. $U_{Z}(x, r)=$ $\{y \in Z: d(x, y)<r\}, x \in Z$. Likewise, $\operatorname{int}(Y), \operatorname{adh}(Y)$ and $\partial(Y), Y \subseteq X$, are respectively used for the topological operators of interior, closure and boundary derived from the metric in $X$, whilst for $Z \subseteq X$, $\operatorname{int}_{Z}(Y)$ and $\partial_{Z}(Y), Y \subseteq Z$, denote the corresponding operators in $Z$ as a metric subspace of $X$. Notice that, for any closed set $Z \subseteq X$, the closure of $Y \subseteq Z$ in the subspace $Z$ coincides with $\operatorname{adh}(Y)$. We first prove the following lemma.

LEMma 2.1. Assume that $\Psi$ satisfies the open set condition for an open set $\mathcal{O} \subseteq X$. Then

(i) $T(E \cap \partial \mathcal{O}) \subseteq E \cap \partial \mathcal{O}$.

(ii) $T(O) \subseteq E \cap \partial \mathcal{O}$.

(iii) Let $G=\operatorname{adh}(\mathbf{O}(T(O)))$. Then $G \subseteq E \cap \partial \mathcal{O}$.

(iv) $T(G) \subseteq G$.

Proof. A proof of (i) can be seen in [11]. We prove it here for completeness. Let $x \in T(E \cap \partial \mathcal{O})$. Then, by (5), there exists a $\psi \in \Psi$ such that $\psi(x) \in E \cap \partial \mathcal{O}$. If $x \in \mathcal{O}$ then $\psi(x) \in \mathcal{O}$, giving a contradiction, which proves (i). Let $x \in T(O)$, and let $\psi \in \Psi$ be such that $\psi(x) \in O$. If $x \in \mathcal{O}$ 
then $\psi(x) \in \psi(\mathcal{O})$ gives $\emptyset \neq \psi(\mathcal{O}) \cap O$, in contradiction with (3). Therefore, $x \in \partial \mathcal{O}$, which proves (ii). Properties (i) and (ii) give $\mathbf{O}(T(O)) \subseteq E \cap \partial \mathcal{O}$. Since $E \cap \partial \mathcal{O}$ is a closed set, property (iii) follows.

Let $x \in T(G)$. Then $\psi_{i}(x) \in G$ for some $i \in M$ and there exists a sequence $y_{k} \rightarrow \psi_{i}(x)$ with $y_{k} \in \mathbf{O}(T(O))$ for all $k \in \mathbb{N}$. There exists a $j \in M$ such that $y_{k} \in E_{j}$ for infinitely many values of $k$. We may assume that $y_{k} \in$ $E_{j}$ for all $k$. If $j \neq i$, then $\psi_{i}(x) \in E_{j} \cap E_{i}$ since $E_{j}$ is closed. Hence $\psi_{i}(x) \in O$, which shows that $x \in T(O) \subseteq G$. If $i=j$, then $\psi_{i}^{-1}\left(y_{k}\right) \in T\left(y_{k}\right) \subseteq \mathbf{O}(T(O))$. Therefore $x=\psi_{i}^{-1}\left(\psi_{i}(x)\right)=\lim _{k} \psi_{i}^{-1}\left(y_{k}\right) \in \operatorname{adh}(\mathbf{O}(T(O)))=G$.

REMARK 2.2. If we follow the lines of the proof of Lemma 2.1, we may check that the properties of the open set $\mathcal{O}$ used there are those in (1) and (3), which are also satisfied by a set $W$ which satisfies the R.O.S.C. From this it follows that the lemma holds if we replace $\mathcal{O}$ with $W$ and the topological operators in $X$ with the corresponding operators in $E$. Observe also that to prove property (2) we only need the properties in (1) and the continuity of the similitudes, so (2) holds for $W$.

Part (iii) of the above lemma shows that the boundary $\partial(\mathcal{O})$ of any open set $\mathcal{O}$ satisfying the O.S.C. contains the set $G$. If a set $W$ satisfies the R.O.S.C., property (iii) of Lemma 2.1 shows that $G \subseteq \partial_{E}(W)$. Therefore the set $V=E-G$ is the largest open set which can satisfy the R.O.S.C. In the next theorem we state that $V$ actually satisfies the R.O.S.C. and we formulate two separation conditions equivalent to the S.O.S.C.

ThEOREM 2.3. The following conditions are equivalent.

(i) The restricted open set condition holds for $\Psi$.

(ii) The open set $V=E-G$, with $G=\operatorname{adh}(\mathbf{O}(T(O)))$, is non-empty.

(iii) The strong open set condition holds for $\Psi$.

We keep the notation for $V$ and $G$ from now onwards. See Example 2.6, Theorem 2.7 and Proposition 2.8 for a discussion of conditions under which we can drop the operator $\operatorname{adh}(\cdot)$ from the definition of $G$.

Proof. (i) $\Rightarrow$ (ii). Assume first that the R.O.S.C. holds for $W$. By Remark 2.2 we know that $G \subseteq \partial_{E}(W)$. Therefore we have $E-G \supseteq E-$ $\partial_{E}(W) \supseteq W \neq \emptyset$.

(ii) $\Rightarrow$ (i). Let $V=E-G$ and assume $V \neq \emptyset$. We denote by $V_{i}$ the set $\psi_{i}(V), i \in M$. If $x \in V$ then $\psi_{i}(x) \in V$, since if we assume on the contrary that $\psi_{i}(x) \in G$ then, by Lemma 2.1(iv), $x \in T(G) \subseteq G$, which is not possible. If $x \in V_{i} \cap O$, then $\psi_{i}^{-1}(x) \in V \cap T(O)$, which is not possible (as $T(O) \subseteq G)$. This shows that $V$ satisfies the R.O.S.C.

(ii) $\Rightarrow$ (iii). Let $V=E-G$ be non-empty. Then $V$ satisfies the R.O.S.C. For every $x \in V$, let

$$
\varrho_{x}=2^{-1} d(x, G) .
$$


Then we claim that the set

$$
\mathcal{O}=\bigcup_{x \in V} U\left(x, \varrho_{x}\right)
$$

which is an open set in $X$, satisfies the S.O.S.C. This will be proved by using the fact that $r_{i} \varrho_{x} \leq \varrho_{\psi_{i}(x)}$ for $x \in V$. To prove this last inequality, we first prove that

$$
U_{E}\left(\psi_{i}(x), 2 r_{i} \varrho_{x}\right) \subseteq V_{i}, \quad x \in V, i \in M .
$$

Observe that, since $E$ and $E_{i}$ are closed sets the topological operators $\operatorname{adh}_{E}(\cdot), \operatorname{adh}_{E_{i}}(\cdot)$ and $\operatorname{adh}(\cdot) \operatorname{coincide.~From~} \operatorname{adh}\left(E_{i}-V_{i}\right) \subseteq \operatorname{adh}\left(E-V_{i}\right)$ it follows that $\partial_{E_{i}}\left(V_{i}\right) \subseteq \partial_{E}\left(V_{i}\right)$. Let $x \in \partial_{E}\left(V_{i}\right)$. Since $x \in \operatorname{adh}\left(E-V_{i}\right)$, either $x \in \operatorname{adh}\left(E_{i}-V_{i}\right)$ or $x \in E_{j}$ for some $j \neq i$. In the first case $\psi_{i}^{-1}(x) \in G$, since $G$ is a closed set. In the second case, $x \in O$, so $\psi_{i}^{-1}(x) \in T(O) \subseteq G$. Therefore $\psi_{i}^{-1}(x) \in G$ in either case, which shows that $\partial_{E}\left(V_{i}\right) \subseteq \psi_{i}(G)$. Since $\psi_{i}: E \rightarrow E_{i}$ is a homeomorphism, we see that $\psi_{i}(G)=\psi_{i}(\partial V)=$ $\partial_{E_{i}}\left(V_{i}\right) \subseteq \partial_{E}\left(V_{i}\right)$, so we have

$$
\partial_{E}\left(V_{i}\right)=\psi_{i}(G) .
$$

From $\emptyset=V_{i} \cap \psi_{i}(G)=V_{i} \cap \partial_{E}\left(V_{i}\right)$ it follows that $V_{i}, i \in M$, is an open set in $E$. If we take a $z$ in $\psi_{i}(G)$ such that $d\left(\psi_{i}(x), \psi_{i}(G)\right)=d\left(\psi_{i}(x), z\right)$, we then have

$$
2 r_{i} \varrho_{x}=r_{i} d(x, G) \leq r_{i} d\left(x, \psi_{i}^{-1}(z)\right)=d\left(\psi_{i}(x), z\right)=d\left(\psi_{i}(x), \partial_{E}\left(V_{i}\right)\right),
$$

which shows that (6) holds.

Assume now that $r_{i} \varrho_{x}>\varrho_{\psi_{i}(x)}$, and let $z \in G$ satisfy $d\left(\psi_{i}(x), z\right)=$ $2 \varrho_{\psi_{i}(x)}$. Then $z \in U_{E}\left(\psi_{i}(x), 2 r_{i} \varrho_{x}\right) \subseteq V_{i} \subseteq V$, which contradicts $z \in G$. Therefore $r_{i} \varrho_{x} \leq \varrho_{\psi_{i}(x)}$. From this, it easily follows that $\psi_{i}(\mathcal{O}) \subseteq \mathcal{O}$.

We now prove that $\psi_{i}(\mathcal{O}) \cap \psi_{j}(\mathcal{O})=\emptyset, i \neq j$. Let $x \in \psi_{i}(\mathcal{O}) \cap \psi_{j}(\mathcal{O})$. From $x \in \psi_{i}(\mathcal{O})$ it follows that there exists a $y \in V$ with $d\left(\psi_{i}(y), x\right)<r_{i} \varrho_{y}$. Analogously, for some $z \in V, d\left(\psi_{j}(z), x\right)<r_{j} \varrho_{z}$. So,

$$
d\left(\psi_{i}(y), \psi_{j}(z)\right)<\max \left\{2 r_{i} \varrho_{y}, 2 r_{j} \varrho_{z}\right\} .
$$

Assume for instance that $r_{j} \varrho_{z} \leq r_{i} \varrho_{y}$. Then using (6) we have

$$
\psi_{j}(z) \in U_{E}\left(\psi_{i}(y), 2 r_{i} \varrho_{y}\right) \subseteq V_{i},
$$

giving the contradiction $\psi_{j}(z) \in V_{i} \cap V_{j}=\emptyset$.

(iii) $\Rightarrow$ (i). If the strong open set condition holds for $\mathcal{O}$, then $V=\mathcal{O} \cap E$ satisfies the R.O.S.C.

REMARK 2.4. Theorem 2.3 shows that each of the separation conditions (i), (ii), and (iii) is equivalent to the O.S.C. for $X=\mathbb{R}^{n}$, since this last condition is equivalent to (iii) in this case (see [14] for a proof). Observe that the logical chain linking the separation conditions (i), (ii) and (iii) 
depends only on the structure of $X$ as a complete metric space. The link between the O.S.C. and the strong open set condition depends on specific properties of $\mathbb{R}^{n}$, as shown by the results by Schief quoted in Section 1.3.

From the proof of the above theorem we can also easily draw the following useful consequence: If $U_{E}(x, r)$ is an open ball contained in $V$, then

$$
\psi_{\mathbf{i}}\left(U_{E}(x, r)\right)=U_{E}\left(\psi_{\mathbf{i}}(x), r_{\mathbf{i}} r\right), \quad \mathbf{i} \in M^{k}, k \in \mathbb{N} .
$$

To see this, we may assume $k=1$ without loss of generality. From $r \leq$ $2 \varrho_{x}$ together with (6), it follows that, if $y \in U_{E}\left(\psi_{i}(x), r_{i} r\right)$, then $y \in$ $U_{E}\left(\psi_{i}(x), 2 r_{i} \varrho_{x}\right) \subseteq V_{i}$, so $\psi_{i}^{-1}(y) \in U_{E}(x, r)$, and $y \in \psi_{i}\left(U_{E}(x, r)\right)$. Therefore $\psi_{i}\left(U_{E}(x, r)\right) \supseteq U_{E}\left(\psi_{i}(x), r_{i} r\right)$. The opposite inclusion is trivial.

REMARK 2.5. In many classical examples of self-similar sets, as the Koch curve, the Sierpinski gasket, the unit cube in $\mathbb{R}^{n}$ as a self-similar set generated by $2^{n}$ similitudes and the twin-dragon fractal, the dynamical boundary $G$ may be written in a simplified form, since $\mathbf{O}(T(O))=\bigcup_{i=1}^{q} T^{i}(O)$ for a finite $q$ in all these cases, and from the fact that, for any closed set $F, T(F)$ is also a closed set, we then get

$$
G=\bigcup_{i=1}^{q} T^{i}(O)
$$

We say that a set $A \subseteq E$ is $T$-finitely generated by a set $B \subseteq E$ if $A \subseteq$ $\bigcup_{i=0}^{q} T^{i}(B)$ for a finite $q$. The following example shows that, in general, $\mathbf{O}(T(O))$ is not $T$-finitely generated by $O$.

EXAMPLE 2.6. Let $\Psi=\left\{\psi_{i}\right\}_{1 \leq i \leq 6}$ be the system of six similitudes mapping the unit square $Q \subseteq \mathbb{R}^{2}$ onto six squares, as indicated in Figure 1. We

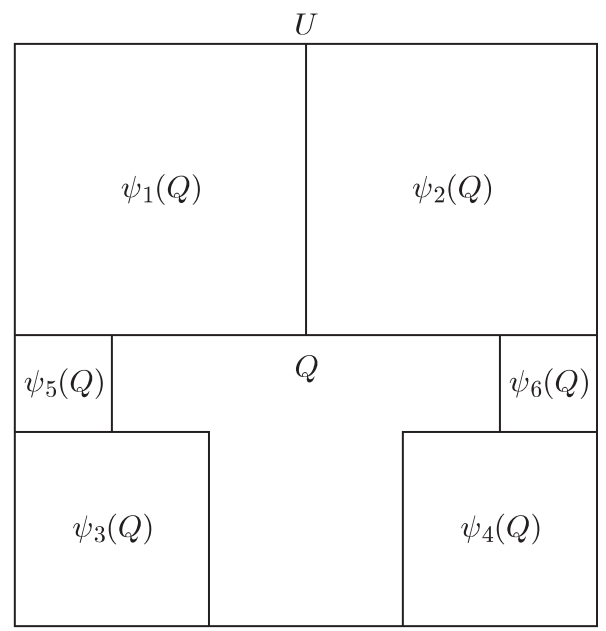

Fig. 1 
assume that the four corners of $Q$ are the fixed points of the similitudes $\psi_{1}, \psi_{2}, \psi_{3}$ and $\psi_{4}$ respectively. The contraction ratios of $\psi_{1}$ and $\psi_{2}$ are $1 / 2$ and the contraction ratios of $\psi_{3}$ and $\psi_{4}$ are $1 / 3$. Thus the upper side $U$ of $Q$ is contained in the self-similar set $E$ generated by $\Psi$, and the lower side of $Q$ contains a ternary Cantor set which is in turn contained in $E$. We now show that $\mathbf{O}(T(O))$ cannot be $T$-finitely generated by $O$ in the sense of Remark 2.5. Let $O_{H}$ denote the horizontal part of the overlapping set, that is, $O_{H}=O-\left(E_{1} \cap E_{2}\right)$. Observe first that $\mathbf{O}\left(T\left(O_{H}\right)\right)$ contains the set of points of $U$ with an $x$-coordinate of the form $2^{k} 3^{-p}$. It is easy to see that the set

$$
\{k \log 2-p \log 3: k, p \in \mathbb{N}\}
$$

is dense in $\mathbb{R}$. This follows from the ergodicity of irrational rotations. Therefore $U \subseteq \operatorname{adh}\left(\mathbf{O}\left(T\left(O_{H}\right)\right)\right) \subseteq G$. Notice also that we have $U \cap \mathbf{O}\left(T\left(O_{H}\right)\right)=$ $U \cap \mathbf{O}(T(O))$, so if $\mathbf{O}(T(O))$ is $T$-finitely generated by $O$ then $U$ is also $T$-finitely generated by $O_{H}$. In this case, taking into account that $\operatorname{dim} Y=$ $\operatorname{dim} T(Y)$ for any $Y \subseteq E$, we get $\operatorname{dim} U=\operatorname{dim} O_{H}$, which gives the contradiction

$$
1=\operatorname{dim} U=\operatorname{dim} O_{H}=\log 2 / \log 3 .
$$

In the following theorem we give a sufficient condition for the boundary of a separating open set to be $T$-finitely generated by the intersection of the separating set with the overlapping set.

THEOREM 2.7. (i) Assume that $\Psi$ satisfies the open set condition for an open set $\mathcal{O}$ with $\mathcal{O} \cap E \neq \emptyset$, and $S \Psi(E \cap \partial \mathcal{O})-\partial \mathcal{O}=O \cap \mathcal{O}$. Then $E \cap \partial \mathcal{O}=\mathbf{O}(T(O \cap \mathcal{O}))$, and $E \cap \partial \mathcal{O}$ is T-finitely generated by $O \cap \mathcal{O}$.

(ii) Assume that $\Psi$ satisfies the strong open set condition and let $V=$ $E-G$. If $S \Psi(G)-G=O \cap V$, then $G=\mathbf{O}(T(O \cap V))$ and $G$ is T-finitely generated by $O \cap V$.

Proof. (i). We first prove that $\bigcup_{i=1}^{k} T^{i}(O \cap \mathcal{O}), k=1,2, \ldots$, are open sets in $E \cap \partial \mathcal{O}$. To see this we prove that if $\mathcal{U}$ is an open set in $E \cap \partial \mathcal{O}$, then $T(O \cap \mathcal{O}) \cup T(\mathcal{U})$ is an open set in $E \cap \partial \mathcal{O}$. Assume that $x \in \mathcal{U} \cup(O \cap \mathcal{O})$. Then $T(x)=\bigcup_{i \in P_{x}} \psi_{i}^{-1}(x)$ for some set of indices $P_{x} \subseteq M$. We treat separately the cases $x \in O \cap \mathcal{O}$ and $x \in \mathcal{U}$.

Assume that $x \in O \cap \mathcal{O}$. Then $U\left(x, \varrho_{x}\right) \cap O \subseteq \mathcal{O}$ for a sufficiently small $\varrho_{x}$. Let $i \in P_{x}$ and $y \in U\left(\psi_{i}^{-1}(x), r_{i}^{-1} \varrho_{x}\right) \cap E \cap \partial \mathcal{O}$. Then $\psi_{i}(y) \in$ $S \Psi(E \cap \partial \mathcal{O}) \cap \mathcal{O} \subseteq O \cap \mathcal{O}$. This shows that

$$
\mathcal{W}_{x}^{i}:=U\left(\psi_{i}^{-1}(x), r_{i}^{-1} \varrho_{x}\right) \cap E \cap \partial \mathcal{O} \subseteq T(O \cap \mathcal{O}) .
$$

Therefore, for every $x \in O \cap \mathcal{O}, T(x)$ is a finite union of points which are interior points of $T(O \cap \mathcal{O})$ in $E \cap \partial \mathcal{O}$. This shows that $T(O \cap \mathcal{O})$ is an open set in $E \cap \partial \mathcal{O}$. 
Assume now that $x \in \mathcal{U}$. Then $U\left(x, \varrho_{x}\right) \cap E \cap \partial \mathcal{O} \subseteq \mathcal{U}$ for a sufficiently small $\varrho_{x}$. We now prove that, for $i \in P_{x}$,

$$
\mathcal{W}_{x}^{i}:=U\left(\psi_{i}^{-1}(x), r_{i}^{-1} \varrho_{x}\right) \cap E \cap \partial \mathcal{O} \subseteq T(O \cap \mathcal{O}) \cup T(\mathcal{U})
$$

Take a $y \in \mathcal{W}_{x}^{i}$. Then $\psi_{i}(y) \in S \Psi(E \cap \partial \mathcal{O})$. If $\psi_{i}(y) \in E \cap \partial \mathcal{O}$ then $\psi_{i}(y) \in \mathcal{U}$, and $y \in T(\mathcal{U})$. If $\psi_{i}(y) \notin E \cap \partial \mathcal{O}$, then $\psi_{i}(y) \in O \cap \mathcal{O}$, and $y \in T(O \cap \mathcal{O})$.

Putting together the two cases considered above, we have proved that, for $x \in(O \cap \mathcal{O}) \cup \mathcal{U}, T(x)$ is a finite union of interior points in $E \cap \partial \mathcal{O}$ of the set $T(O \cap \mathcal{O}) \cup T(\mathcal{U})$, which shows that $T(O \cap \mathcal{O}) \cup T(\mathcal{U})$ is open in $E \cap \partial \mathcal{O}$. If we apply inductively this fact to $\mathcal{U}=\bigcup_{i=1}^{k} T^{i}(O \cap \mathcal{O}), k=1,2, \ldots$, we see that all these sets are open in $E \cap \partial \mathcal{O}$.

We prove that $E \cap \partial \mathcal{O}=\bigcup_{i=1}^{q} T^{i}(O \cap \mathcal{O})$ for a finite $q$. The compact set $H=E \cap \partial \mathcal{O}-\bigcup_{i=0}^{\infty} T^{i}(O \cap \mathcal{O})$ satisfies $S \Psi(H) \subseteq H$. This follows from the fact that, otherwise, for some $x \in H$ and some $\psi \in \Psi$, either $\psi(x) \in O \cap \mathcal{O}$, which gives the contradiction $x \in T(O \cap \mathcal{O})$, or $\psi(x) \in \bigcup_{i=1}^{\infty} T^{i}(O \cap \mathcal{O})$, which gives the contradiction $x \in T\left(\bigcup_{i=1}^{\infty} T^{i}(O \cap \mathcal{O})\right) \subseteq \bigcup_{i=0}^{\infty} T^{i}(O \cap \mathcal{O})$. Therefore the compact set $K=\bigcap_{i=1}^{\infty} S \Psi(H)$ satisfies $S \Psi(K)=K$. Then, by the uniqueness of $E$, either $K$ is an empty set or $K=E$. Since $K=E$ would imply $H=E$, and so $E \cap \mathcal{O}=\emptyset$, we see that $K$ is an empty set, so $H$ is also empty. The proof of (i) then follows from the compactness of $E \cap \partial \mathcal{O}$.

(ii) The proof of (i) remains valid if we replace $\mathcal{O}$ with $V$ and $E \cap$ $\partial \mathcal{O}$ with $G$. Notice that in this case $V \cap E \neq \emptyset$ is guaranteed by Theorem 2.3 .

Notice that one of the hypotheses of this theorem holds in each case mentioned in Remark 2.5. For the twin-dragon fractal and the unit cube, the O.S.C. is satisfied for an open set in $\mathbb{R}^{n}$ contained in $E$. In the following proposition we see that the hypotheses of Theorem 2.7 follow from this fact.

Proposition 2.8. If $\Psi$ satisfies the strong open set condition and $\mathcal{O}:=$ $\operatorname{int}(E) \neq \emptyset$, then the following properties hold: $V=\mathcal{O}, G=\partial E=\partial V$, $S \Psi(\partial E)-\partial E=O \cap V, V$ satisfies the hypotheses of Theorem 2.7 and $\partial E$ is $T$-finitely generated by $T(O)$.

Pr o of. We first check that $\mathcal{O}$ satisfies the O.S.C. From the bicontinuity of $\psi_{i}, i \in M$, it follows that $\psi_{i}(\mathcal{O})=\psi_{i}(\operatorname{int}(E))=\operatorname{int}\left(E_{i}\right) \subseteq \mathcal{O}$. If $\psi_{i}(\mathcal{O}) \cap$ $\psi_{j}(\mathcal{O}) \neq \emptyset, i \neq j$, then $O$ has a non-empty interior, so it contains some cylinder set. It follows that $T^{q}(O)=E$ for a finite $q$, so $G=E$ and by Theorem 2.3, $\Psi$ cannot satisfy the S.O.S.C. This shows that $\mathcal{O}$ satisfies the O.S.C. and it also satisfies the R.O.S.C. Since $\mathcal{O}$ is the largest open set contained in $E$, it has to coincide with $V$. If $x \in S \Psi(\partial E)-\partial E$ then 


$$
\begin{aligned}
& x \in \psi_{i}(\partial E)-\partial E=\partial\left(E_{i}\right) \cap \mathcal{O} \text { for some } i \in M . \text { Therefore } \\
& x \in \operatorname{adh}\left(X-E_{i}\right) \cap \mathcal{O}=\left(\left(\bigcup_{j \neq i} E_{j}\right) \cup \operatorname{adh}(X-E)\right) \cap \mathcal{O}=\left(\bigcup_{j \neq i} E_{j}\right) \cap \mathcal{O},
\end{aligned}
$$

which proves that $x \in O \cap \mathcal{O}$, and the hypotheses of Theorem 2.7 hold.

3. Dimension of the overlapping set. Given $F \subseteq E$, for every $k \in \mathbb{N}$ consider the set $\mathcal{F}_{k}=\left\{\mathbf{i} \in M^{k}: E_{\mathbf{i}} \cap F \neq \emptyset\right\}$ of codes and denote by $\langle F\rangle_{k}$ the self-similar set generated by the system $\left\{\psi_{\mathbf{i}}: \mathbf{i} \in \mathcal{F}_{k}\right\}$ of similitudes. It turns out that the sequence of self-similar sets $\langle F\rangle_{k}$ gives a useful information about the dimension of the set $F$ under certain hypotheses. This is the method used in this section to obtain estimates for the dimension of the overlapping set. We first prove the following lemma.

Lemma 3.1. Let $F$ be a T-invariant subset of E. Then

$$
F \subseteq \bigcap_{k \in \mathbb{N}}\langle F\rangle_{k}
$$

If $F$ is closed but not necessarily T-invariant, then

$$
F \supseteq \bigcap_{k \in \mathbb{N}}\langle F\rangle_{k} .
$$

Proof. Assume that $F$ is $T$-invariant, and let $x \in F$ and $k \in \mathbb{N}$. Then, for any natural number $q$ and any $\mathbf{i} \in \pi^{-1}(x), \pi\left(\sigma^{q k}(\mathbf{i})\right) \in T^{q k}(x) \subseteq F$, so $E_{\mathbf{j}(k)} \cap F \neq \emptyset$ for $\mathbf{j}=\sigma^{q k}(\mathbf{i})$. Therefore $x \in\langle F\rangle_{k}$, which proves $(7)$.

Assume now that $F$ is closed. Let $\delta_{k}=\max \left\{\left|E_{\mathbf{i}}\right|: \mathbf{i} \in \mathcal{F}_{k}\right\}$ and let

$$
[F]_{\delta_{k}}=\left\{x \in E: d(F, x) \leq \delta_{k}\right\} .
$$

Then

$$
\langle F\rangle_{k} \subseteq \bigcup_{\mathbf{i} \in \mathcal{F}_{k}} E_{\mathbf{i}} \subseteq[F]_{\delta_{k}} .
$$

Since $\delta_{k}$ tends to zero as $k$ tends to infinity, we see that

$$
\bigcap_{k \in \mathbb{N}}\langle F\rangle_{k} \subseteq \bigcap_{k \in \mathbb{N}}[F]_{\delta_{k}}=F .
$$

Falconer [3] defines a subself-similar set as a compact set $F \subseteq E$ such that $F \subseteq S \Psi(F)$. It may be easily seen that $F \subseteq E$ is subself-similar if and only if $F$ is closed and $T$-invariant (see [3] for a proof). Using the properties of subself-similar sets obtained in the quoted reference, we prove

Lemma 3.2. Assume that $X=\mathbb{R}^{n}$.

(i) If $F$ is a $T$-invariant subset of $E$, then

$$
\overline{\operatorname{dim}}_{\mathrm{B}} F \leq \inf \left\{\operatorname{dim}\langle F\rangle_{k}: k \in \mathbb{N}\right\},
$$


where $\overline{\operatorname{dim}}_{\mathrm{B}} Y$ stands for the upper box dimension of $Y \subseteq \mathbb{R}^{n}$ (see $[2,9]$ for a definition).

(ii) Assume that $\Psi$ satisfies the O.S.C. If $F$ is a closed T-invariant set, then

$$
\operatorname{dim} F=\overline{\operatorname{dim}}_{\mathrm{B}} F=\lim _{k \rightarrow \infty} \operatorname{dim}\langle F\rangle_{k} .
$$

The upper box dimension is not less than either the Hausdorff, the packing and the lower box dimensions (see [17, 2, 9] for definitions). Hence, if (i) holds, we may replace $\overline{\operatorname{dim}}_{\mathrm{B}}(\cdot)$ in (i) with any of the above mentioned dimensions and the inequality still holds. The Hausdorff dimension is a lower bound for the lower box, upper box and packing dimensions [17, 2, 9]. Therefore, if (ii) holds, all these dimensions coincide.

Proof of Lemma 3.2. It is well known $[2,9]$ that the upper box and the Hausdorff dimensions of a self-similar set coincide. Hence $\overline{\operatorname{dim}}_{\mathrm{B}}\langle F\rangle_{k}=$ $\operatorname{dim}\langle F\rangle_{k}$, and (i) is then a trivial consequence of (7).

To prove (ii) we use the fact that, since $F$ is a subself-similar set, we have (see [3])

$$
\operatorname{dim} F=\sup \left\{t>0: \sum_{k \in \mathbb{N}} \sum_{\mathbf{i} \in \mathcal{F}_{k}} r_{\mathbf{i}}^{t}=\infty\right\}
$$

Assume that $t<\lim \sup _{k} \operatorname{dim}\langle F\rangle_{k}$. Then $t<\operatorname{dim}\langle F\rangle_{k}$ for infinitely many values of $k$, and for such $k$ 's we know that $\sum_{\mathbf{i} \in \mathcal{F}_{k}} r_{\mathbf{i}}^{t}>1$. Therefore $\operatorname{dim} F \geq t$, and so $\operatorname{dim} F \geq \lim \sup _{k} \operatorname{dim}\langle F\rangle_{k}$.

We may now prove the main result of this section.

THEOREM 3.3. Assume that $X=\mathbb{R}^{n}$ and that $\Psi$ satisfies the strong open set condition for the open set $\mathcal{O} \subseteq \mathbb{R}^{n}$. Then

$$
\begin{aligned}
\overline{\operatorname{dim}}_{\mathrm{B}} O & \leq \operatorname{dim} \operatorname{adh}(\mathbf{O}(T(O)))=\lim _{k \rightarrow \infty} \operatorname{dim}\langle\operatorname{adh}(\mathbf{O}(T(O)))\rangle_{k} \\
& \leq \operatorname{dim} E \cap \partial \mathcal{O}=\lim _{k \rightarrow \infty}\langle E \cap \partial \mathcal{O}\rangle_{k}<s .
\end{aligned}
$$

Pr o o f. For $Y \subseteq E$, we have $Y \subseteq S \Psi(T(Y))$, which proves that $\overline{\operatorname{dim}}_{\mathrm{B}}(O)$ $\leq \overline{\operatorname{dim}}_{\mathrm{B}}(T(O))$ by the invariance of $\overline{\operatorname{dim}}_{\mathrm{B}}$ under similitudes. Since $T(O) \subseteq$ $\operatorname{adh}(\mathbf{O}(T(O)))$, the first inequality in (8) follows. The next equality follows from Lemmas 2.1(iv) and 3.2(ii). Lemma 2.1(iii) gives the next inequality. The subsequent equality in (8) is a consequence of Lemmas 2.1(i) and 3.2(ii).

Let $x \in \mathcal{O} \cap E$, and $\mathbf{i} \in \pi^{-1}(x)$. Then $E_{\mathbf{i}(k)} \subseteq \mathcal{O} \cap E$ for $k$ large enough, so $E_{\mathbf{i}(k)} \cap \partial \mathcal{O}=\emptyset$. Let $\mathcal{F}_{k}=\left\{\mathbf{i} \in M^{k}: E_{\mathbf{i}} \cap \partial \mathcal{O} \neq \emptyset\right\}$. Then $M^{k}-\mathcal{F}_{k} \neq \emptyset$, and therefore $\sum_{\mathbf{i} \in \mathcal{F}_{k}} r_{\mathbf{i}}^{s}<\sum_{\mathbf{i} \in M^{k}} r_{\mathbf{i}}^{s}=1$, which shows that $\inf \left\{\operatorname{dim}\langle E \cap \partial \mathcal{O}\rangle_{k}: k \in \mathbb{N}\right\}<s$, and Lemmas 2.1(i) and 3.2(i) yield the last inequality in (8). 
Notice that the string (8) remains true for any open set $\mathcal{O} \subseteq E$ satisfying the R.O.S.C. and also for every open set $\mathcal{O} \subseteq \mathbb{R}^{n}$ satisfying the O.S.C. except for the last inequality.

Acknowledgements. I am grateful to Christoph Bandt, Kenneth Falconer and Pertti Mattila for their helpful comments on this paper.

\section{References}

[1] C. Bandt and S. Graff, Self-similar sets 7. A characterization of self-similar fractals with positive Hausdorff dimension, Proc. Amer. Math. Soc. 114 (1992), 9951001 .

[2] J. K. Falconer, Fractal Geometry, Wiley, Chichester, 1990.

[3] —, Sub-self-similar sets, Trans. Amer. Math. Soc. 347 (1995), 3121-3129.

[4] J. Feder, Fractals, Plenum Press, New York, 1988.

[5] J. E. Hutchinson, Fractals and self-similarity, Indiana Univ. Math. J. 30 (1981), 713-747.

[6] T. Lindstrøm, Brownian motion on nested fractals, Mem. Amer. Math. Soc. 420 (1990).

[7] J. Kigami and M. Lapidus, Weyl's problem for the spectral distribution of Laplacians on P.C.F. self-similar sets, Commun. Math. Phys. 158 (1993), 93-125.

[8] B. Mandelbrot, The Fractal Geometry of Nature, Freeman, San Francisco, 1977.

[9] P. Mattila, Geometry of Sets and Measures in Euclidean Spaces, Cambridge Univ. Press, 1995.

[10] M. Morán, Multifractal components of multiplicative functions, preprint.

[11] M. Morán and J. M. Rey, Singularity of self-similar measures with respect to Hausdorff measures, Trans. Amer. Math. Soc. 350 (1998), 2297-2310.

[12] - - - Geometry of self-similar measures, Ann. Acad. Sci. Fenn. Math. 22 (1997), 365-386.

[13] P. A. P. Moran, Additive functions of intervals and Hausdorff measures, Proc. Cambridge Philos. Soc. 42 (1946), 15-23.

[14] A. Schief, Separation properties for self-similar sets, Proc. Amer. Math. Soc. 122 (1994), 111-115.

[15] -, Self-similar sets in complete metric spaces, ibid. 121 (1996), 481-489.

[16] S. Stella, On Hausdorff dimension of recurrent net fractals, ibid. 116 (1992), 389400.

[17] C. Tricot, Two definitions of fractal dimension, Math. Proc. Cambridge Philos. Soc. 91 (1982), 57-74.

Departamento de Análisis Económico

Universidad Complutense de Madrid

Campus de Somosaguas

28223 Madrid, Spain

E-mail: ececo06@sis.ucm.es 